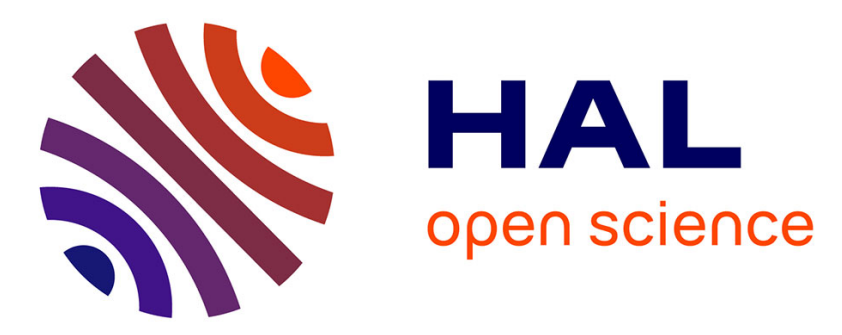

\title{
Composantes de la dépense énergétique au cours du nycthémère chez la truie adulte à l'entretien : rôle de l'activité physique
}

J. Noblet, X.S. Shi, S. Dubois

\section{- To cite this version:}

J. Noblet, X.S. Shi, S. Dubois. Composantes de la dépense énergétique au cours du nycthémère chez la truie adulte à l'entretien : rôle de l'activité physique. Productions Animales, 1994, 7 (2), pp.135-142. hal-00896082

\section{HAL Id: hal-00896082 https://hal.science/hal-00896082}

Submitted on 1 Jan 1994

HAL is a multi-disciplinary open access archive for the deposit and dissemination of scientific research documents, whether they are published or not. The documents may come from teaching and research institutions in France or abroad, or from public or private research centers.
L'archive ouverte pluridisciplinaire HAL, est destinée au dépôt et à la diffusion de documents scientifiques de niveau recherche, publiés ou non, émanant des établissements d'enseignement et de recherche français ou étrangers, des laboratoires publics ou privés. 
INRA Prod. Anim., 1994, 7 (2), 135 - 142.
J. NOBLET, X.S. SHI, S. DUBOIS

INRA Station de Recherches Porcines 35590 Saint Gilles
Composantes de la dépense énergétique au cours du nycthémère chez la truie adulte à l'entretien : rôle de l'activité physique

En production porcine, le besoin d'énergie correspondant à la couverture des dépenses énergétiques d'entretien représente de $50 \%$ chez le porc en croissance, à plus de $90 \%$ chez la truie gravide, du besoin énergétique total. De surcroît, dans le dernier cas, le besoin est extrêmement variable selon les conditions d'environnement et, au sein d'un même troupeau, entre les animaux. Il en résulte des niveaux de performances (gain pondéral et reproduction) très variables, le syndrôme de la «truie maigre» en constituant une illustration extrême. La quantification des principales composantes de la dépense énergétique d'entretien représente une voie d'analyse de cette variabilité.

La dépense énergétique d'entretien correspond d'abord à la somme de la production de chaleur à jeun et de l'extra-chaleur d'utilisation de l'aliment ingéré, ces deux quantités étant mesurées chez un animal au repos et en conditions de thermoneutralité. S'y ajoutent une dépense d'énergie liée à l'activité phy-

\section{Résumé}

La production de chaleur (HP, par calorimétrie indirecte) et l'activité physique (station debout vs station couchée) de six truies Large White castrées (poids vif moyen : $208 \mathrm{~kg}$ ), maintenues individuellement en chambres respiratoires, à une température de $24^{\circ} \mathrm{C}$, et alimentées une fois par jour à 100,60 ou $0 \%$ de leur besoin énergétique d'entretien ont été mesurées en continu après le repas. Chaque truie a fait l'objet de 8 à 10 séries de mesures sur des régimes différents au cours des 9 mois de l'expérience. L'analyse des données permet de quantifier les différentes composantes de la production de chaleur totale chez des animaux à l'entretien. En moyenne, pour les 6 truies de l'étude, le coût énergétique de la station debout (240 minutes par jour) est de $14,9 \mathrm{~kJ}$ par minute de station debout, ce qui représente $15 \%$ de la production de chaleur totale $\left(400 \mathrm{~kJ} / \mathrm{kg} \mathrm{P}^{0,75}\right)$. L'activité physique est enregistrée exclusivement pendant la période diurne. La production de chaleur pour un niveau d'activité physique nul $\left(340 \mathrm{~kJ} / \mathrm{kg} \mathbf{P}^{0,75}\right)$ se répartit entre la production de chaleur à

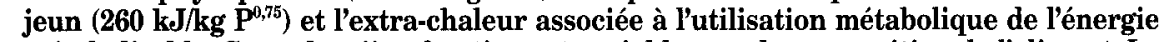
métabolisable. Cette dernière fraction est variable avec la composition de l'aliment. Le coût énergétique de la prise d'aliment est évalué à $2 \%$ de la quantité d'énergie métabolisable ingérée. Les résultats mettent également en évidence que cette répartition de la production de chaleur varie au cours du nycthémère. Combinées aux données de la bibliographie, notre étude met en évidence que le besoin en énergie et le niveau des performances de la truie gravide sont directement dépendants des conditions de logement et de son comportement. sique et, en fonction des conditions d'environnement, des dépenses de thermorégulation. Dans le cas particulier de la truie adulte (vide ou gravide), le niveau d'activité physique varie avec le mode de logement (bâtiment fermé : en individuel ou en groupe ; plein air...) et, au sein d'un même troupeau, en fonction de paramètres tels que l'état corporel ou le passé nutritionnel (Cariolet et Dantzer 1984, Robert et al 1992). Le comportement de stéréotypie (durée de la station debout très prolongée, mordillement des barres ou de l'auge...) manifesté par la truie à l'attache représente une illustration extrême de cette variabilité. Par ailleurs, en raison du faible niveau d'alimentation pratiqué en dehors de la période de lactation et de la réduction de l'isolation thermique résultant de la diminution de ses réserves corporelles, la truie adulte est sensible aux conditions de milieu, sa température critique étant voisine de $22^{\circ} \mathrm{C}$ lorsqu'elle est élevée individuellement à l'attache (Noblet et al 1989). En pratique, il existe alors de nombreuses situations induisant des dépenses de thermorégulation importantes chez la truie.

Les effets de la température ambiante sur la dépense énergétique de la truie adulte élevée individuellement ont été analysés par Noblet et al (1989 et 1990) qui concluaient à un besoin en EM supplémentaire voisin 
de $800 \mathrm{~kJ}$ par ${ }^{\circ} \mathrm{C}$ d'abaissement de la température ambiante au-dessous de la température critique. Dans cet article, nous nous attacherons à l'analyse des autres composantes de la dépense énergétique d'entretien, en insistant plus particulièrement sur l'activité physique. Les résultats sont pour la plupart issus d'une étude dont l'objectif principal était l'analyse du rendement d'utilisation de l'énergie métabolisable (EM) pour l'entretien chez le porc (Noblet et al 1993a). Le modèle animal choisi est la truie adulte ovariectomisée (afin de s'affranchir des éventuels effets du cycle sexuel), alimentée de façon à couvrir sa dépense énergétique d'entretien et maintenue en cage à métabolisme. Dans ce cas, l'activité physique correspond essentiellement à la station debout, comparativement à la station couchée. Des détails complémentaires sur l'aspect activité physique de cette étude sont donnés par ailleurs (Noblet et al 1993b).

\section{1 / Conditions expérimentales}

L'expérience est basée sur 6 truies ovariectomisées adultes de race Large White pesant en moyenne $208 \mathrm{~kg}$. Elles reçoivent pendant 8 à 10 périodes consécutives de 28 jours une quantité d'aliment correspondant à la couverture de leur dépense énergétique d'entretien (415 kJ d'EM par $\mathrm{kg} \mathrm{P}^{0,75}$ ). Chaque truie reçoit 8 à 10 des 14 régimes (un régime par période) de l'étude. Des ajustements individuels de l'apport alimentaire sont réalisés périodiquement de façon à maintenir constants pendant toute l'expérience le poids vif et l'épaisseur de gras dorsal de chaque animal. Au cours de chaque période, la truie est adaptée à un nouveau régime pendant 17 jours et placée ensuite pendant 11 jours dans une cage permettant la collecte des fèces et de l'urine. Pendant les 8 derniers jours de la période, la cage est introduite dans une chambre respiratoire afin de mesurer la consommation d'oxygène et les productions de gaz carbonique et de méthane. Deux niveaux d'alimentation sont appliqués dans la chambre respiratoire : $100 \%$ (niveau 100) de la dépense énergétique d'entretien pendant les 5 premiers jours et $60 \%$ (niveau 60) les 3 derniers jours. Au niveau 60, le premier jour de mesure, considéré comme une période d'adaptation ou de transition, n'est pas pris en compte dans les calculs ultérieurs de production de chaleur. De plus, à 3 ou 4 reprises au cours de l'expérience, les échanges respiratoires sont mesurés sur chaque truie pendant 48 heures, un seul repas étant distribué au début de la mesure. Le deuxième jour de mesure peut être considéré comme une période de jeûne (niveau 0). Pendant toute l'expérience, les truies sont éclairées de 8 h00 à 20 h00 et la température ambiante est maintenue à $24^{\circ} \mathrm{C}$.

Les 14 régimes de l'étude, dont la composition et les caractéristiques nutritionnelles sont rapportées par Noblet et al (1993c) sont de type complexe, à base de blé, amidon de maïs, tourteau de soja, farine de viande, son de blé, remoulages de blé, pulpes de betterave, «corn gluten feed», paille de blé, mélasse de canne et huile de colza. Les teneurs moyennes en énergie digestible (ED) et en EM des 14 régimes sont respectivement 15,6 et 14,4 MJ par $\mathrm{kg}$ de matière sèche (MS) ingérée. La ration quotidienne est apportée sous forme de granulés et en un seul repas à 8 h30. Au niveau 100 , la quantité de MS ingérée est en moyenne de $1580 \mathrm{~g}$ par jour. Les animaux disposent d'eau à volonté. La durée du repas est enregistrée à 2 ou 3 reprises par régime et par truie au cours de la période d'adaptation au régime.

Les échanges respiratoires quotidiens sont calculés par intervalles successifs de 7 minutes ; le résultat prend en compte les modifications de la concentration de gaz dans la chambre respiratoire au cours de l'intervalle. La production de chaleur est calculée à partir des échanges respiratoires, pour chaque intervalle et par journée, selon la formule de Brouwer (1965). La durée et la fréquence de la station debout sont également mesurées en continu et calculées pendant les mêmes intervalles de 7 minutes, à l'aide de faisceaux infra-rouges situées à l'avant et à l'arrière de la cage.

Les contributions respectives de l'activité physique (AHP pour «activity heat production») et du métabolisme au repos (RHP pour «resting heat production») à la production de chaleur totale (HP) ont été estimées au niveau 100 pour chaque truie recevant un régime à l'aide d'une équation de régression dans laquelle la production de chaleur (par intervalle de 7 minutes) est une fonction linéaire du temps après le repas ( $\mathrm{t}$ en minutes) et de la durée de l'activité physique (activ), exprimée en pour cent de la durée de l'intervalle de mesure. Dans le modèle qui s'écrit alors : HP (par minute $)=a+b x t+c \times$ activ, a représente $\mathrm{RHP}$ au début du repas, $b$, la variation (diminution) de RHP par minute après le début du repas, et c la dépense moyenne d'énergie associée à la station debout (ou AHP, par minute). Les données des trois premiers intervalles de la journée situés après le début du repas ne sont pas considérés dans le calcul du fait qu'ils incluent la prise d'aliment. La valeur de RHP pour chaque journée de mesure correspond à la différence entre la production de chaleur totale qui est mesurée et AHP, ellemême égale à c multiplié par la durée quotidienne de la station debout. La démarche utilisée est illustrée à la figure 1. La valeur de c obtenue lorsque l'animal est au niveau 100 est appliquée aux journées de mesure réalisées aux niveaux d'alimentation 60 et 0 .

La production de chaleur au jeûne (ou FHP pour "Fasting Heat Production») de chaque truie est. supposée constante pendant toute l'expérience et calculée, indépendamment du régime qu'elle reçoit, à partir de la relation entre RHP aux niveaux 100 et 60 et les quantités d'EM correspondantes. La valeur de FHP (pour un niveau d'activité physique nul) est obtenue par extrapolation de RHP à une quantité d'EM ingérée nulle. Quant à l'extra-chaleur associée à l'utilisation de l'EM (ou TEF pour "Thermic Effect of Feed»), elle correspond, pour une truie recevant un régime, à la différence entre RHP au niveau 100 et FHP. 
Figure 1. Variations de la production de chaleur et de la durée de la station debout (\% du temps) au cours d'une période de 24 heures suivant un repas (exemple d'une journée de mesure).

Production de chaleur $(\mathrm{kJ} / \mathrm{min})$

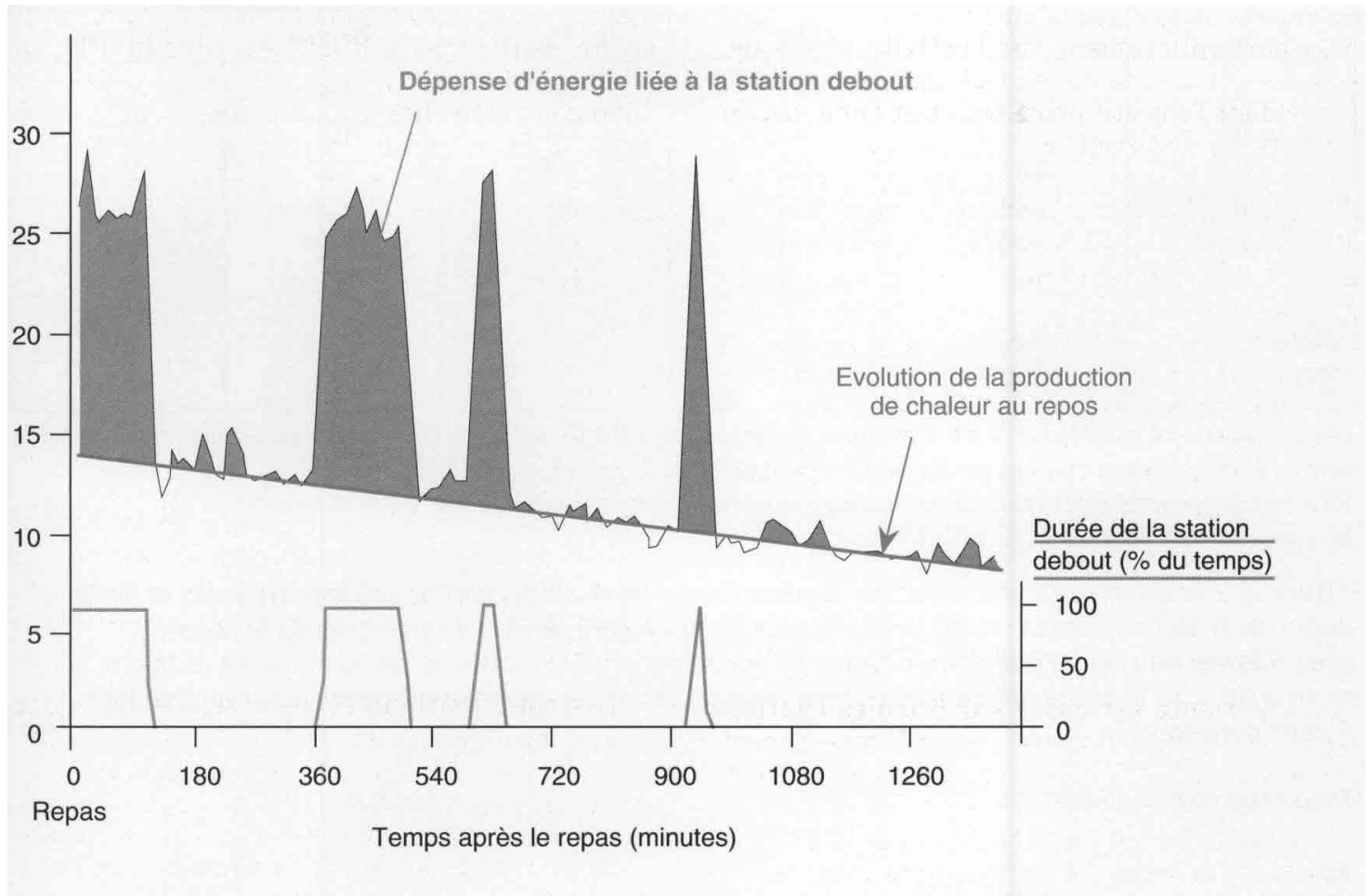

La valeur de EM ingérée considérée (ou EMc) dans le calcul de FHP ou TEF correspond à la quantité d'EM réellement ingérée diminuée de AHP (MJ/jour). Des détails complémentaires sur la méthode de calcul de FHP et TEF sont rapportés par Noblet et al (1993a).

De façon à décrire l'évolution de la production de chaleur totale, de l'activité physique et des composantes de la production de chaleur au cours du nycthémère au niveau 100 , les données d'une journée sont divisées en 24 séquences successives, la première durant 20 minutes et incluant la totalité du temps de repas, alors que les suivantes ont une durée de 60 minutes. Les résultats d'une truie recevant un régime correspondent à la moyenne des résultats des 5 jours de mesure au niveau d'alimentation 100 .

Les résultats obtenus sur chaque truie à chaque période de mesure $(n=56)$ ont été soumis à une analyse de variance avec comme effets principaux le numéro de la truie $(n=6)$ et le régime $(n=14)$, selon un dispositif en blocs incomplets équilibrés. Pour ce qui concerne l'analyse de l'évolution nycthémérale de HP et ses composantes et de l'activité physique, l'effet séquence $(n=24)$ est également considéré. Pour la plupart des critères analysés, l'effet du régime est significatif (Noblet et al 1993a). Les résultats rapportés dans les tableaux 1,2 et 4 correspondent par conséquent aux moyennes ajustées pour les différences entre régimes. SAS (1988) a été utilisé pour l'ensemble des analyses statistiques.

\section{2 / Résultats et discussion}

Au niveau 100 , la quantité d'aliment consommé par repas est en moyenne de $1580 \mathrm{~g}$ de matière sèche. La durée de consommation du repas est relativement constante (environ 13 minutes), à l'exception de la truie D (18 minutes) (tableau 1). La vitesse d'ingestion peut alors être évaluée à environ $120 \mathrm{~g}$ MS par minute, soit $2 \mathrm{~g}$ par minute et par $\mathrm{kg} \mathrm{P}^{0,75}$. Ce résultat est proche de celui obtenu par Dourmad (1989) avec des truies primipares en lactation ( $90 \mathrm{~g}$ par minute). Selon les résultats de Nienaber et al (1990), la vitesse d'ingestion serait plus faible chez le porc en croissance (moins de $1 \mathrm{~g}$ par minute et par $\mathrm{kg} \mathrm{P}^{0,75}$ ).

Conformément au protocole, la valeur moyenne de HP des 6 truies de l'étude est presqu'identique à la quantité d'EM ingérée (tableau 1). Il en résulte que HP correspond pratiquement à la quantité d'EM pour la couverture des besoins énergétiques d'entretien $(\mathrm{EMm})$. Exprimé par $\mathrm{kg}$ de poids métabolique $\left(\mathrm{kg} \mathrm{P}^{0,75}\right)$ et corrigé pour la différence entre EM ingérée et $\mathrm{HP}(0,25 \mathrm{~kJ}$ de HP par $\mathrm{kJ}$ de différence : Noblet et al 1993a), $\mathrm{EM}_{\mathrm{m}}$ est équivalent à $400 \mathrm{~kJ}$ par kg $\mathrm{P}^{0,75}$, soit une valeur légèrement inférieure à celles obtenues (environ $420 \mathrm{~kJ} / \mathrm{kg} \mathrm{P} \mathrm{P}^{0,75}$ ) avec des truies gravides adultes (Noblet et al 1989c) ou en première gestation (Close et al 1985, Noblet et Etienne 1987), alimentées de façon plus libérale. Conformément aux conclusions de Mac Leod et al (1979) tirées de résultats obtenus sur coqs adultes, la différence entre les études précitées et la présente expérience peut résulter d'une adaptation métabolique au faible niveau d'ali- 
Tableau 1. Poids vif (PV), énergie métabolisable (EM) ingérée, production de chaleur (HP) et durée (minutes par jour) de la station debout et du repas chez 6 truies adultes à l'entretien.

\begin{tabular}{|c|c|c|c|c|c|}
\hline \multirow[t]{2}{*}{ Truie } & \multirow{2}{*}{$\begin{array}{c}\text { PV moyen } \\
\text { (kg) }\end{array}$} & \multirow{2}{*}{ 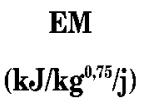 } & \multirow{2}{*}{$\begin{array}{c}\text { HP } \\
\left(\mathbf{k J} / \mathbf{k g}^{0,75 / \mathrm{j})}\right.\end{array}$} & \multicolumn{2}{|c|}{ Durée } \\
\hline & & & & Debout & Repas \\
\hline A & $216^{c}$ & $404^{b}$ & $404^{\mathrm{ab}}$ & $284^{\mathrm{bc}}$ & $13,0^{\mathrm{a}}$ \\
\hline B & $210^{d}$ & $415^{\mathrm{ab}}$ & $384^{\mathrm{c}}$ & $100^{\mathrm{d}}$ & $13,2^{\mathrm{a}}$ \\
\hline $\mathrm{C}$ & $226^{b}$ & $423^{\mathrm{a}}$ & $388^{\text {he }}$ & $244^{c}$ & $13,4^{\mathrm{a}}$ \\
\hline $\mathrm{D}$ & $232^{\mathrm{a}}$ & $416^{\mathrm{ab}}$ & $402^{\mathrm{ab}}$ & $153^{\mathrm{d}}$ & $18,2^{\mathrm{b}}$ \\
\hline $\mathrm{E}$ & $175^{\mathrm{f}}$ & $424^{\mathrm{a}}$ & $419^{a}$ & $323^{\mathrm{ab}}$ & $13,1^{\mathrm{a}}$ \\
\hline $\mathrm{F}$ & $191^{\mathrm{e}}$ & $410^{\mathrm{ab}}$ & $418^{a}$ & $360^{\mathrm{a}}$ & $13,7^{\mathrm{a}}$ \\
\hline Moyenne & 208 & 415 & 402 & 241 & 14,2 \\
\hline ETR & 4 & 14 & 18 & 65 & 2,3 \\
\hline
\end{tabular}

Chaque donnée est la moyenne de 8 à 10 mesures, correspondant à 8 à 10 régimes différents. Dans une colonne, les valeurs suivies de la même lettre ne sont pas significativement différentes $(P>0,05)$.

ETR : écart type résiduel de l'analyse de variance qui prend en compte les effets truie $(n=6)$ et régime $(n=14)$.

Figure 2. Evolution de la production de chaleur totale, de la production de chaleur au repos et de la durée de la station debout (\% du temps) au cours d'une période de 24 heures après le repas, chez 6 truies adultes à l'entretien. Chaque valeur représente la moyenne de 56 mesures (6 truies recevant 8 à 10 régimes différents), chaque mesure étant elle-même la moyenne obtenue pendant 5 jours consécutifs.

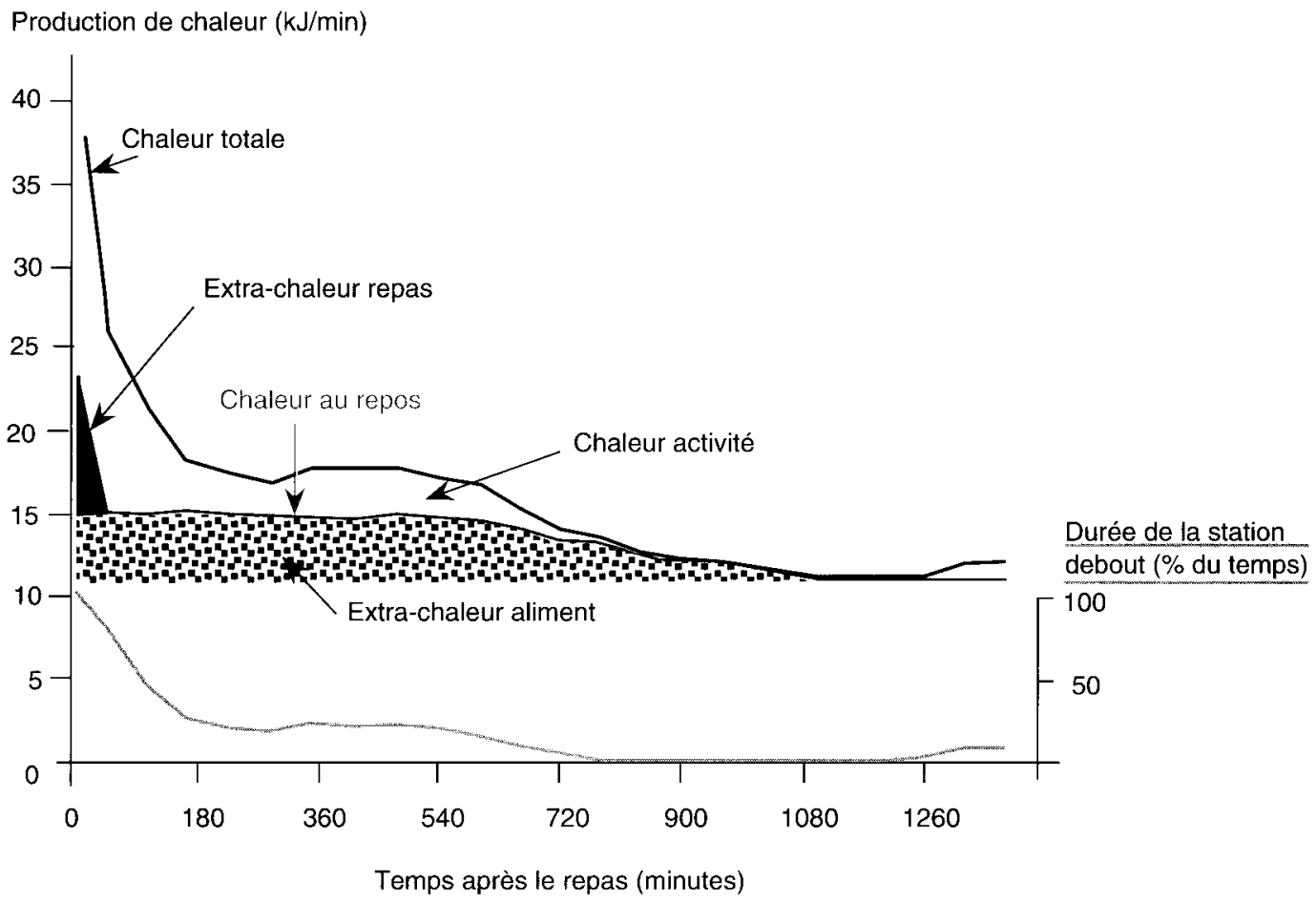

mentation des animaux de notre étude. Enfin, $\mathbf{E M}_{\mathrm{m}}$ n'est pas constant puisque l'écart entre les valeurs extrêmes obtenues sur les 6 animaux de l'étude (380 à $\left.420 \mathrm{~kJ} / \mathrm{kg} \mathrm{P}^{0,75}\right)$ représente environ $10 \%$ de la valeur moyenne.

La durée moyenne de la station debout est de 241 minutes par jour avec des écarts particulièrement importants entre les animaux (tableau 1). A l'inverse, pour chaque truie, les durées de la station debout mesurées au cours des périodes successives (8 à 10) de l'étude sont comparables $(P>0,05)$, ce qui signifie que le niveau d'activité physique est caractéris- tique de chaque animal. La durée moyenne obtenue dans notre étude sur un faible nombre d'animaux et dans des conditions d'habitat particulières est tout à fait comparable à celle mesurée dans des élevages, sur des truies attachées (Cariolet et Dantzer 1984). Dans des conditions d'habitat comparables à celles de la présente étude, nous avions mesuré un niveau d'activité plus faible (160 minutes) chez des truies adultes alimentées de façon plus libérale (Noblet et al 1989). En accord avec les observations de Robert et al (1992), la différence entre nos deux études peut résulter de l'écart de niveau d'alimentation. De même, Cariolet 
Tableau 2. Dépenses d'énergie au repos $(R H P)$ et dépense associée à la station debout (AHP) chez 6 truies adultes à l'entretien (HP : production totale de chaleur).

\begin{tabular}{|c|c|c|c|c|c|}
\hline \multirow[t]{2}{*}{ Animal } & \multirow{2}{*}{$\underset{\mathbf{k J} / \mathbf{k g}^{0,75}}{\mathbf{R H P}}$} & \multicolumn{4}{|c|}{ AHP } \\
\hline & & $\mathrm{kJ} / \mathrm{min}$ & $\mathrm{kJ} / \mathrm{min} / \mathrm{kg}^{0,75}$ & $\mathrm{~kJ} / \mathrm{j} / \mathrm{kg}^{0,75}$ & $\% \mathrm{HP}$ \\
\hline A & $341^{b}$ & $12,9^{d}$ & $0,228^{c}$ & $69,0^{\mathrm{b}}$ & $15,7^{b}$ \\
\hline B & $352^{\text {ab }}$ & $19,0^{\mathrm{a}}$ & $0,345^{\mathrm{a}}$ & $28,0^{\mathrm{d}}$ & $8,1^{\mathrm{c}}$ \\
\hline $\mathrm{C}$ & $326^{c}$ & $15,2^{\text {bc }}$ & $0,259^{b c}$ & $59,0^{\mathrm{b}}$ & $15,6^{b}$ \\
\hline $\mathrm{D}$ & $360^{\mathrm{a}}$ & $16,0^{\mathrm{b}}$ & $0,269^{b}$ & $42,7^{\mathrm{cd}}$ & $10,2^{c}$ \\
\hline $\mathrm{E}$ & $328^{\mathrm{bc}}$ & $13,4^{\text {cd }}$ & $0,278^{b}$ & $92,1^{\mathrm{a}}$ & $21,3^{\mathrm{a}}$ \\
\hline F & $328^{\mathrm{bc}}$ & $12,7^{\mathrm{d}}$ & $0,248^{b c}$ & $87,9^{a}$ & $21,2^{a}$ \\
\hline Moyenne & 340 & 14,9 & 0,273 & 62,3 & 15,2 \\
\hline ETR & 14 & 1,9 & 0,034 & 16,0 & 3,3 \\
\hline
\end{tabular}

Voir le tableau 1 pour le nombre d'observations et les analyses statistiques.

Tableau 3. Dépense énergétique associée à la station debout (AHP) dans différentes espèces.

\begin{tabular}{|c|c|c|c|c|}
\hline \multirow[t]{2}{*}{ Espèce } & \multirow{2}{*}{$\begin{array}{l}\text { Poids vif } \\
(\mathbf{k g})\end{array}$} & \multicolumn{2}{|c|}{ AHP } & \multirow[t]{2}{*}{ Auteurs } \\
\hline & & $\mathrm{kJ} / \mathbf{k g} / \mathbf{1 0 0} \mathrm{min}$ & $\mathrm{kJ} / \mathrm{kg}^{\mathrm{N}, 75} / 100 \mathrm{~min}$ & \\
\hline Porc & $\begin{array}{r}27 \\
40 \\
75 \\
177 \\
208\end{array}$ & $\begin{array}{r}13,3 \\
11,8 \\
8,7 \\
8,7 \\
7,2\end{array}$ & $\begin{array}{l}30,2 \\
29,7 \\
25,6 \\
31,8 \\
27,3\end{array}$ & $\begin{array}{l}\text { Hörnicke } 1970 \\
\text { McDonald et al } 1988 \\
\text { Susenbeth et Menke } 1991 \\
\text { Cronin et al } 1986 \\
\text { Noblet et al } 1993\end{array}$ \\
\hline $\begin{array}{l}\text { Mouton } \\
\text { Bœuf } \\
\text { Poulet } \\
\text { Rat }\end{array}$ & $\begin{array}{r}57 \\
63 \\
273\end{array}$ & $\begin{array}{l}2,3 \\
2,3 \\
0,9 \\
7,0 \\
2,4\end{array}$ & $\begin{array}{l}6,4 \\
6,6 \\
3,8 \\
8,3 \\
2,4\end{array}$ & $\begin{array}{l}\text { Osuji } 1974 \\
\text { Toutain et al } 1977 \\
\text { Vercoe } 1973 \\
\text { MacLeod et Jewitt } 1985 \\
\text { Keller et Janji } 1991\end{array}$ \\
\hline
\end{tabular}

et Dantzer (1984) suggéraient un niveau d'activité motrice plus élevé chez des truies recevant de faibles apports alimentaires ou amaigries. Cependant, cette observation ne serait valable qu'après une période prolongée puisque les durées de la station debout dans notre étude sont équivalentes aux niveaux 100 et 60 . Au-delà de la variabilité individuelle et des effets liés au niveau alimentaire ou à l'état des truies, il peut subsister des écarts de niveau d'activité physique entre élevages associés au logement et à la conduite du troupeau.

La figure 2 indique clairement que l'activité motrice se manifeste essentiellement au cours de la première moitié de la journée $(90 \%$ au cours des 11 premières heures). Au cours de la première séquence de 20 minutes qui correspond au repas, les truies passent $100 \%$ de leur temps dans la station debout. Ce rapport diminue progressivement dans les heures suivantes pour atteindre une valeur moyenne de $16 \%$ qui persiste jusqu'à la 11 ème heure après le repas. Pendant le reste du nycthémère qui correspond à la nuit, le niveau d'activité physique est pratiquement nul. Ces observations sont en accord avec celles de Cariolet et Dantzer (1984), ces auteurs signalant également que l'activité motrice est fortement liée à la distribution de l'aliment.
La production de chaleur associée à la station debout (AHP) est en moyenne de 14,9 kJ par minute, la valeur la plus élevée $(19,0 \mathrm{~kJ}$ par minute) étant obtenue avec la truie $B$ pour laquelle la durée de la station debout est la plus faible (tableau 2). Comme pour la durée de la station debout, les valeurs de AHP mesurées sur une truie au cours des périodes successives $(n=8$ à 10$)$ sont similaires $(P>0,05)$. Les différences entre truies sont fortement atténuées lorsque AHP est exprimée par kg de poids métabolique : si l'on excepte la truie $B$, AHP est voisin de $0,26 \mathrm{~kJ}$ par minute et par $\mathrm{kg} \mathrm{P}^{0,75}$. Ce résultat est proche de celui que l'on peut estimer à partir des données de Cronin et al (1986) chez des truies gravides $\left(0,30 \mathrm{~kJ} / \mathrm{min} / \mathrm{kg} \mathrm{P}^{0,75}\right)$. Il est par contre plus élevé que la première estimation obtenue par Noblet et al (1989) $\left(0,20 \mathrm{~kJ} / \mathrm{min} / \mathrm{kg} \mathrm{P}^{0,75}\right)$, également chez la truie adulte gravide mais avec une approche moins rigoureuse. Comme l'indique le tableau 3 , les données obtenues chez la truie sont comparables à celles estimées chez le porc en croissance par Hornicke (1970) ou McDonald et al (1988). Mais les données de ce tableau montrent également que le coût en énergie de la station debout est nettement plus élevé chez le porc que pour les autres espèces considérées.
La production de chaleur associée à la station debout est en moyenne de $15 \mathrm{~kJ} / \mathrm{min}$. Elle représente $15 \%$ de la production totale de chaleur sur le nycthémère. 
Tableau 4. Energie métabolisable (EM) ingérée et composantes de la production de chaleur totale (MJ/jour) chez 6 truies adultes à l'entretien.

\begin{tabular}{|c|c|c|c|c|c|}
\hline \multirow[t]{2}{*}{ Truie } & \multirow{2}{*}{$\begin{array}{c}\text { EM } \\
\text { ingérée }\end{array}$} & \multicolumn{4}{|c|}{ Production de chaleur } \\
\hline & & AHP & RHP & FHP & TEF \\
\hline A & $20,84^{\mathrm{c}}$ & $4,41^{\text {a }}$ & $15,93^{\mathrm{c}}$ & 12,21 & $3,71^{\mathrm{b}}$ \\
\hline B & $21,11^{\mathrm{c}}$ & $4,46^{\mathrm{a}}$ & $16,87^{\mathrm{d}}$ & 13,22 & $3,65^{b}$ \\
\hline $\mathrm{C}$ & $22,78^{\mathrm{b}}$ & $1,53^{\circ}$ & $19,44^{b}$ & 14,65 & $4,79^{\mathrm{a}}$ \\
\hline D & $23,07^{\mathrm{b}}$ & $3,88^{\text {ab }}$ & $19,11^{\mathrm{ab}}$ & 14,35 & $4,76^{\mathrm{a}}$ \\
\hline $\mathrm{E}$ & $24,65^{\mathrm{a}}$ & $3,42^{\mathrm{bc}}$ & $19,00^{b}$ & 14,90 & $4,10^{\mathrm{ab}}$ \\
\hline F & $24,69^{\mathrm{a}}$ & $2,53^{\mathrm{d}}$ & $21,34^{\mathrm{a}}$ & 16,70 & $4,64^{\mathrm{a}}$ \\
\hline Moyenne & 22,74 & 3,34 & 18,64 & 14,36 & 4,27 \\
\hline ETR & 0,97 & 0,93 & 0,97 & & 0,81 \\
\hline
\end{tabular}

Voir le tableau 1 pour le nombre d'observations et les analyses statistiques

AHP : production de chaleur liée à l'activité, RHP : production de chaleur au repos, FHP : production de chaleur à jeun,

TEF : extra-chaleur de l'aliment ingéré.

En relation avec les différences de durée de la station debout entre les truies, AHP, calculée sur l'ensemble du nycthémère, varie de 28 à $90 \mathrm{~kJ} / \mathrm{kg} \mathrm{P}^{0,75}$, pour une valeur moyenne de $62 \mathrm{~kJ} / \mathrm{kg} \mathrm{P}^{0,75}$. A'HP représente alors en moyenne $15 \%$ de la production de chaleur totale, ce rapport variant, selon les animaux, de 8 à $21 \%$ (tableau 2). La valeur moyenne du rapport est comparable à celle calculée par Verstegen et al (1987) chez la truie, mais inférieure à celle estimée par Halter et al (1980) pour des porcelets. A l'inverse, elle est plus faible chez le porc en croissance maintenu dans des conditions similaires ( $7 \%$ selon J. Noblet et al, non publié).

La production de chaleur au repos (RHP), calculée comme la différence entre la production de chaleur totale et AHP, est en moyenne de $340 \mathrm{~kJ} / \mathrm{kg} \mathrm{P}^{0,75} / \mathrm{j}$, soit, pour les animaux de notre étude, $13,2 \mathrm{~kJ}$ par minute et par truie (tableau 2). La comparaison de ce résultat avec le coût moyen en énergie de la station debout $(14,9 \mathrm{~kJ} / \mathrm{min})$ montre que la station debout, comparativement à la position couchée, se traduit par un doublement de la dépense d'énergie de l'animal.

La figure 2 montre que RHP n'est pas constante au cours de la journée : la valeur est maximale et relativement constante $(14,8$ $\mathrm{kJ} / \mathrm{min}$ ) au cours des séquences 2 à 11 et décroît progressivement au cours de la nuit pour être minimale à la fin du nycthémère $(11,0 \mathrm{~kJ} / \mathrm{min})$. Cette valeur minimale de RHP, équivalente à environ $290 \mathrm{~kJ}$ par $\mathrm{kg} \mathrm{P}^{0,75}$ et par jour, peut être considérée comme une première estimation de la production de chaleur au jeûne. La différence entre RHP $(13,2 \mathrm{~kJ} / \mathrm{min}$ pour les séquences 2 à 24 ) et la valeur minimale de RHP mesurée à la fin du nycthémère $(11,0 \mathrm{~kJ} / \mathrm{min})$ peut être considérée comme une estimation de l'extra-chaleur d'utilisation de l'EM ingérée. Cette différence cumulée sur l'ensemble de la journée est alors égale à environ 3,35 MJ par jour, soit 17\% de l'EM ingérée (EMc).
Au cours de la première séquence du nycthémère qui inclut le repas, la valeur de RHP est très supérieure à celle calculée pendant les 10 séquences suivantes ( 22,6 vs $14,8 \mathrm{~kJ} / \mathrm{min}$ ). L'écart entre les deux valeurs $(7,8 \mathrm{~kJ} / \mathrm{min})$ peut être considéré comme le coût énergétique de la prise d'aliment et de l'activité physique supplémentaire qui y est associé (figure 2). Pour l'ensemble de la séquence, ceci représente une dépense d'énergie de $156 \mathrm{~kJ}(7,8 \times 20 \mathrm{~min})$, soit environ $100 \mathrm{~kJ}$ par $\mathrm{kg}$ de MS ingérée. Si l'on inclut le coût énergétique obligatoire de la station debout pendant le repas $(14,9 \mathrm{~kJ} / \mathrm{min})$, le coût énergétique total de la prise d'aliment est alors voisin de $450 \mathrm{~kJ}$ au cours de la séquence 1 , soit $285 \mathrm{~kJ}$ par $\mathrm{kg}$ de MS ingérée ou $2 \%$ de la quantité d'EM ingérée.

La production de chaleur mesurée au-delà de 24 heures après le repas (niveau 0) n'est pas constante et continue de décroître : la valeur minimale de RHP obtenue entre 40 et 48 heures après le dernier repas sur les truies de notre étude est égale, en moyenne, à $260 \mathrm{~kJ} / \mathrm{kg} \mathrm{P}^{0,75} / \mathrm{j}$, soit $30 \mathrm{~kJ} / \mathrm{kg} \mathrm{P}^{0,75} / \mathrm{j}$ de moins que la valeur de RHP mesurée 24 heures après un repas. L'extrapolation à EM ingérée nulle de l'équation de régression entre RHP et EM ingérée $(\mathrm{EMc})$ conduit à une estimation de FHP également voisine de $260 \mathrm{~kJ} / \mathrm{kg}^{0,75} / \mathrm{j}$ pour les 6 truies. Cette dernière estimation de FHP a été retenue pour le calcul de TEF (Noblet et al 1993a).

A partir des données moyennes présentées au tableau 4, TEF représente alors en moyenne $23 \%$ de l'EM ingérée. En d'autres termes, le rendement d'utilisation $(\mathrm{km})$ de l'EM pour la couverture des dépenses d'entretien est de $77 \%$ chez le porc. Mais, comme le montrent Noblet et al (1993a), ce rendement varie avec la composition de l'aliment ( 74 à $81 \%$ pour les 14 régimes de l'étude). Ces variations résultent de différences de rendement d'utilisation de l'EM des éléments digestibles : 100, 80, 70 et $60 \%$ respectivement pour les matières grasses, l'ensemble amidon + sucres, les 
matières azotées et les parois végétales. Il faut également noter que les valeurs de TEF ou $\mathrm{k}_{\mathrm{m}}$ sont dépendantes du mode d'estimation de FHP. Ainsi, dans notre étude, l'adoption de RHP mesurée 24 heures après le repas comme estimation de FHP conduit à une estimation de TEF représentant $17 \%$ de EMc, soit une valeur de $\mathrm{k}_{\mathrm{m}}$ voisine de $83 \%$. Cette méthode est d'ailleurs comparable à celle utilisée en nutrition humaine dans laquelle RHP et TEF sont mesurés successivement à l'issue d'une nuit de jeûne.

Les différentes composantes de la production de chaleur de la truie adulte à l'entretien sont rassemblées au tableau 4. De façon résumée, la dépense d'énergie liée à l'activité physique représente en moyenne $15 \%$ du total, mais cette proportion est variable selon les animaux (tableau 2). L'essentiel (65\%) de la production de chaleur provient de la dépense énergétique minimale mesurée chez l'animal à jeun. L'extra-chaleur associée à l'utilisation de l'EM équivaut en moyenne à $19 \%$ de la production de chaleur totale ou à $23 \%$ de celle mesurée pour un niveau d'activité physique nulle ; cette proportion est susceptible de varier avec la composition chimique de l'aliment.

A partir de nos résultats (Noblet et Shi 1993, Noblet et al 1993a), il est également possible de décrire l'utilisation de l'énergie brute ingérée par la truie adulte à l'entretien (figure 3 ). Les pertes d'énergie au niveau fécal représentent en moyenne $15 \%$ de l'énergie brute ingérée pour l'ensemble des 14 régimes, cette fraction étant variable avec essentiellement la teneur en parois végétales de l'aliment. La production de méthane $(1,1 \%$ de l'énergie brute ingérée) est également fonction des caractéristiques du régime et, plus particulièrement, de la teneur en parois végétales dégradables par fermentation dans le gros intestin. Les pertes d'énergie dans l'urine, relativement élevées par rapport au porc en croissance, sont à associer à l'important excès de protéines dans l'aliment ( $20 \%$ par rapport à la MS), relativement aux besoins de truies à l'entretien. Conformément au dispositif expérimental, la quantité d'énergie fixée est très faible, de sorte que la production de chaleur représente une quantité équivalente à la quantité d'EM ingérée.

Cette répartition de l'énergie brute ingérée ou de la production de chaleur qui a été mesurée dans les conditions de l'entretien sera notablement modifiée par le niveau d'alimentation, toute variation de la quantité d'aliment consommé à partir de celle permettant de couvrir les dépenses d'entretien entraînant une variation de la composante extra-chaleur (environ $0,25 \mathrm{~kJ}$ par $\mathrm{kJ}$ d'EM) et de la quantité d'énergie fixée $(0,75 \mathrm{~kJ}$ par kJ d'EM).

\section{Conclusions}

Les résultats de notre étude ont permis de quantifier les composantes de la dépense énergétique d'entretien, et plus spécifiquement le coût en énergie de la station debout chez la
Figure 3. Répartition de l'énergie brute ingérée entre l'énergie fixée et les pertes d'énergie dans les fèces, les urines, le méthane et les composantes de la production de chaleur, chez des truies adultes à l'entretien (moyenne de 56 valeurs obtenues avec 6 truies recevant chacune 8 à 10 régimes).

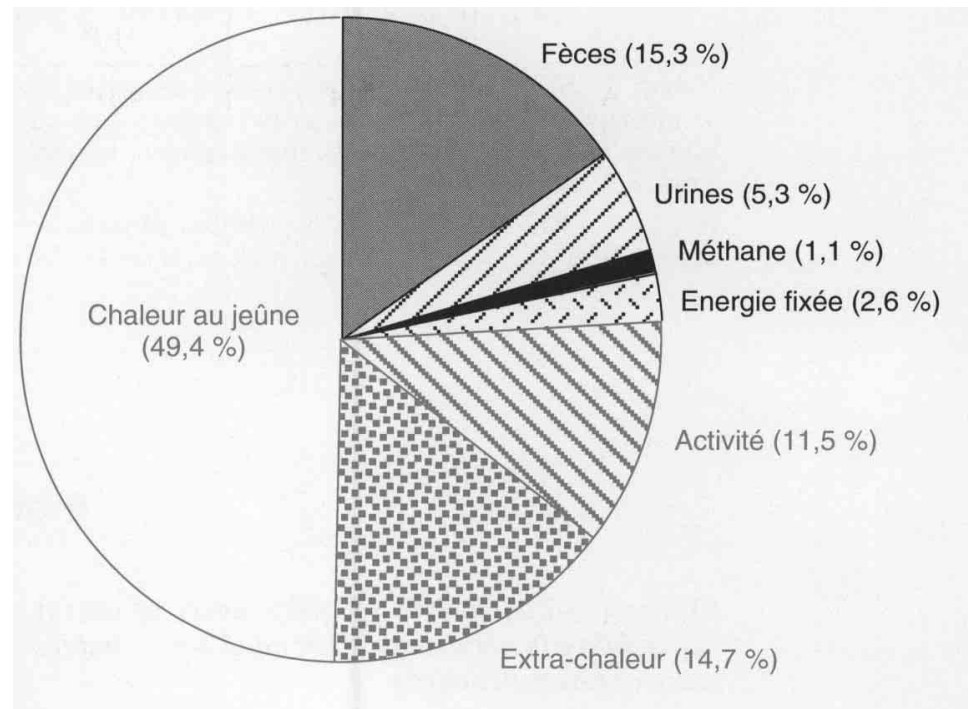

truie. Ce dernier représente l'essentiel du coût énergétique de l'activité motrice dans la plupart des conditions d'élevage. La valeur obtenue $(15 \mathrm{~kJ} / \mathrm{min})$ apparait élevée par rapport à celle d'autres espèces. Il en résulte un coût total qui peut représenter jusqu'à $25 \%$ de la dépense énergétique totale chez des truies particulièrement actives (plus de 400 minutes de station debout par jour) et, par voie de conséquence, une réduction de la quantité d'énergie disponible pour la constitution des réserves corporelles chez la truie gravide. A titre d'illustration, une augmentation de 100 minutes de la durée de la station debout équivaut à une dépense énergétique supplémentaire de $170 \mathrm{MJ}$ sur l'ensemble d'une gestation et une diminution concomitante de $3,5 \mathrm{~kg}$ du gain de lipides, soit 10 à $15 \%$ de la masse adipeuse totale de la truie.

Par ailleurs, le moindre dépôt de gras et la réduction de la quantité d'énergie disponible au-delà de la couverture des dépenses obligatoires d'entretien (et de croissance de l'utérus chez la femelle gestante) entraîne une élévation de la température critique et des dépenses supplémentaires de thermorégulation et, si l'on se réfère aux résultats de Cariolet et Dantzer (1984), une élévation concomitante de l'activité physique. Cet enchaînement d'événements place l'animal dans un "cercle vicieux" qui, à lui seul, rend compte du syndrôme de la "truie maigre", qui a été abondamment décrit, ou des écarts de performances au sein d'un même troupeau. Une correction des apports alimentaires doit par conséquent être appliquée pour prendre en compte ces effets combinés et interdépendants de l'activité physique, de l'environnement climatique, de l'état corporel et du niveau alimentaire chez la truie gravide.
Une augmentation de 100 min de la durée quotidienne de station debout équivaut à une baisse de 3,5 kg du gain de lipides sur l'ensemble d'une gestation. 


\section{Publications originales utilisées pour la préparation de cet article}

\section{(la liste des autres références peut être obtenue auprès des auteurs)}

Noblet J., Shi X.S., 1993. Comparative digestibility of energy and nutrients in growing pigs fed ad libitum and adult sows fed at maintenance. Livest. Prod. Sci., 34, 137-152.

Noblet J., Shi X.S., Dubois S., 1993a. Metabolic utilization of dietary energy and nutrients for maintenance energy requirements in sows: basis for a net energy system. Br. J. Nutr., 70, 407-419.

Noblet J, Shi X.S., Dubois S., 1993b. Energy cost of standing activity in sows. Livest. Prod. Sci., 34, 127 136.

\section{Summary}

Nycthemeral variations of components of energy expenditure in maintenance fed adult sows; importance of physical activity.

Heat production (HP, indirect calorimetry) of six Large White ovariectomized sows (mean BW : $208 \mathrm{~kg}$ ), kept individually in metabolism cages at $24^{\circ} \mathrm{C}$ and fed once a day (d) at 100,60 and $0 \%$ of their maintenance energy level and their physical activity (standing vs lying) were recorded continuously over 24 hours after a meal. Each sow was measured 8 to 10 times with 8 to 10 different diets over the 9 months experiment. Data were used to quantify the components of HP in maintenance fed sows. On average for the 6 sows, the energy cost of standing activity (240 minutes per day) was $14,9 \mathrm{~kJ} / \mathrm{min}$ standing, which represents $15 \%$ of total daily HP (400 kJ/ $\left.\mathrm{kg}^{0,75}\right)$. Standing activity occurred only during the diurnal part of the day. Resting heat production $\left(340 \mathrm{~kJ} / \mathrm{kg}^{0,75}\right)$ originated from fasting heat production $\left(260 \mathrm{~kJ} / \mathrm{kg}^{0,75}\right)$ and the heat increment of metabolizable energy intake. This last component is affected by the chemical characteristics of the diet. The importance of each component of HP varies over the 24 hours period after a meal. Combined with literature data, our results suggest that energy requirements and performance of pregnant sows are highly dependent on environmental conditions and their behaviour.

NOBLET J., SHI X.S., DUBOIS S., 1994. Composantes de la dépense énergétique au cours du nycthémère chez la truie adulte à l'entretien : rôle de l'activité physique. INRA Prod. Anim., 7 (2), 135-142. 